\title{
Tribological modelling of spherical bearings with complex spherical-based geometry and motion
}

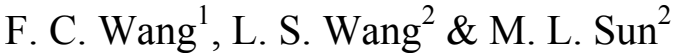 \\ ${ }^{I}$ Wuhan University of Science and Technology, China \\ ${ }^{2} \mathrm{ZWZ}$ (Wafangdian Bearing Group Corporation), China
}

\begin{abstract}
A general tribological simulation system for the study of spherical bearings with complex spherical-based geometry and under operating conditions was developed in order to simulate friction, lubrication and dynamic contact mechanics, as well as wear on the basis of the regular spherical-grid-data-model (SGDM), where the bearing surface was assumed as being perfectly spherical. The elastic deformation of the complex spherical bearing was evaluated by the equivalent discrete spherical convolution model (EDSC) and the spherical fast Fourier transform technique (SFFT). The representation of either macro- or micro-geometries of spherical bearings in motion and the corresponding simulation of the tribological complexity were performed by the fixed-tracked method (FTM) in the simulation system. Several examples of numerical experiments of transient elastohydrodynamic lubrication, wear prediction and friction, as well as dynamic contact mechanics of spherical bearings with nonsphericity, were investigated in order to validate the tribological simulation system of spherical or aspheric bearings. It was found that the complex tribological problems of the bearings could be resolved by the general methodologies developed in the simulation system.

Keywords: spherical-grid-data-model (SGDM), fixed-tracked method (FTM), equivalent discrete spherical convolution (EDSC), spherical fast Fourier transform (SFFT), spherical bearing, aspheric bearing, non-sphericity, tribological simulation system, elastohydrodynamic lubrication (EHL), mixed lubrication, friction, contact mechanics, wear prediction.
\end{abstract}




\section{Introduction}

Spherical bearings have wide applications in industrial engineering and bioengineering, such as spherical bushing, hip joint replacements and spherical joints (Wang et al. [1] and Flores et al. [2]). The significant progress of tribological modelling of spherical bearings, including friction, wear and lubrication, has been achieved in the past decade ([1] and Wang et al. [3]). Most of the simulation works have been carried out by the regular spherical-grid-datamodel (SGDM), where the bearing surface is assumed as being perfectly spherical. However, the geometry of a real spherical bearing is complex, which is introduced by the global or local non-sphericity of bearing surfaces in manufacturing, such as machining marks and roughness, and by the modification of bearing geometry due to wear, as well as possible optimisation design, such as texture or non-spherical bearing surface, [1]. The geometry of the real spherical bearing can be described by the geometrical variations relative to the nominal spherical surface. Additionally, the non-sphericity of bearings under the given operating condition can cause complex tribological problems, for example both the dynamic contact mechanics and the computational wear prediction of spherical bearings with a hard-on-hard bearing material combination under the multi-directional transient motion and dynamic loading condition need to consider the geometrical modifications of two bearing surfaces due to wear with time. Therefore, accurate modelling for the study of the complex tribological problems is helpful to understand the tribological mechanism of the real spherical or aspheric bearing.

The aim of this study was to develop a tribological simulation system for the study of the complex tribological problems of the real spherical or aspheric bearing with complex bearing geometry on the basis of the spherical-grid-datamodel. Two cases of spherical and non-spherical bearings under the multidirectional transient motion and dynamic loading condition were simulated to validate the simulation system. It was hoped that the general methods with the developed in-house software in the simulation system could be applied to obtain the satisfactory solutions of the complex tribological problems under the given operating conditions, including friction, lubrication and mixed lubrication, dynamic contact mechanics and wear.

\section{Bearing geometry and representation}

The geometry of a real spherical bearing or spherical bushing can be described as shown in figs. 1(a) and 1(b). The applied motion and loading to the bearing are described in the appropriate spherical coordinate system, as shown in figs. 2 and 3. When deformation is considered, the complex geometry of a real spherical or aspheric bearing can be described by the geometrical components with the different scales as follows:

$$
r_{i}=r_{i 0}(\varphi, \theta)+\Delta r_{i}(\varphi, \theta)+\Theta_{i}(\varphi, \theta)+\delta_{i}(\varphi, \theta)
$$


where the subscript $r_{0}$ represents the radius of nominal spherical bearing surface. The non-spherical variations of an aspheric bearing can be represented in the spherical coordinates by the deviations, $\Delta r_{i}$, relative to the nominal spherical surface, as shown in figs. 4(a) and 4(b), while $\Theta_{i}$ is the micro-geometrical change such as roughness or machining marks, relative to the overall smooth bearing geometry such as ellipsoidal bearing surface. The elastic deformation of bearing can be evaluated by the equivalent discrete spherical convolution model (EDSC) combined with the corresponding spherical fast Fourier transform (SFFT), [1] and Wang et al. [4].

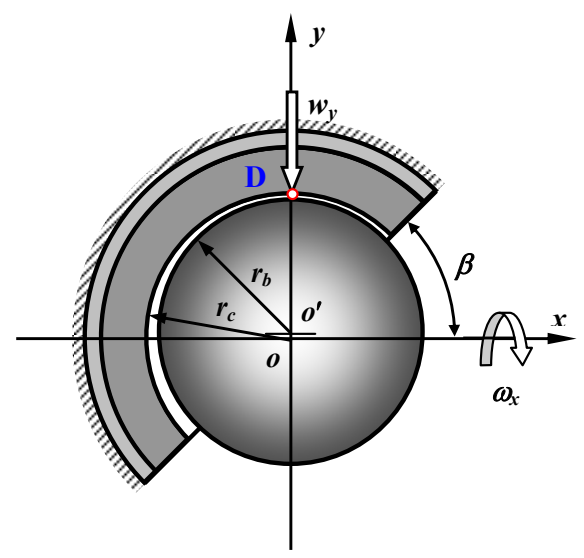

(a)

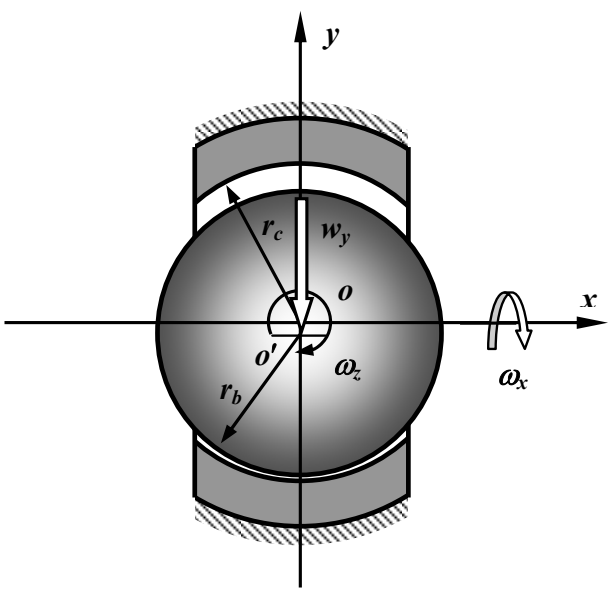

(b)

Figure 1: $\quad$ Geometry model of spherical bearing (a) and spherical bushing (b). 


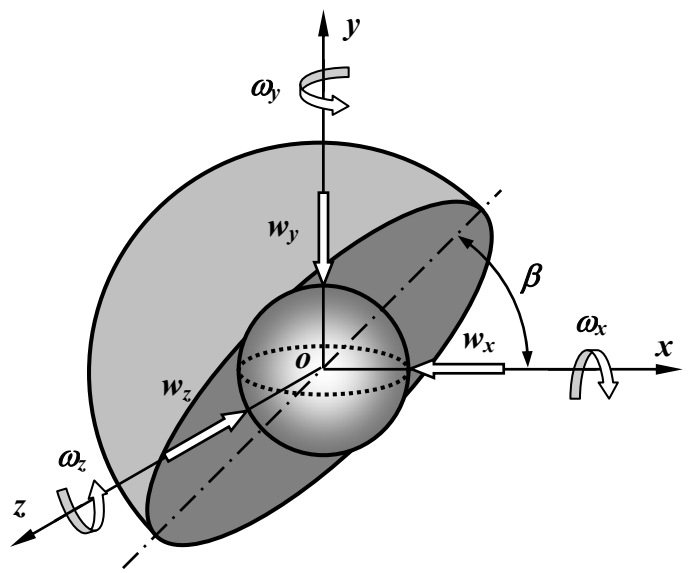

Figure 2: $\quad$ Spherical bearing under loading and motion.

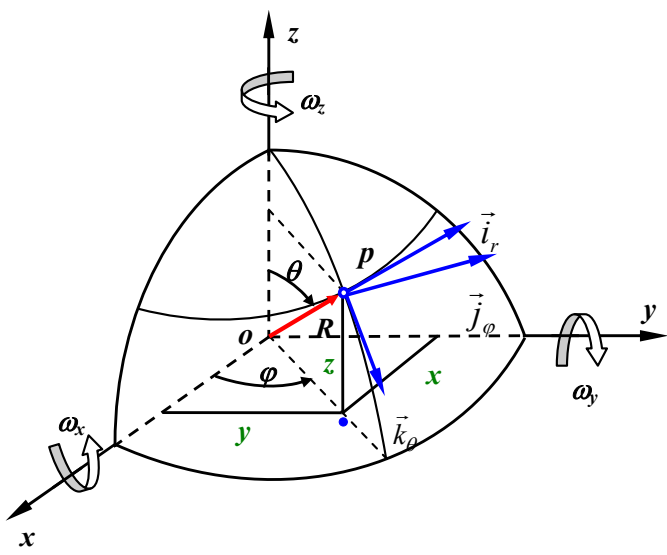

Figure 3: Spherical coordinate system.

Real bearing surfaces need to be accurately described by the discrete spherical mesh-grid space in spherical coordinate system during simulations, for example the geometry representation of complex or moving bearing surfaces is required for the tribological modelling such as the transient lubrication of aspheric bearing and the computational wear modelling of spherical bearing with the geometry modification. For such problems, the fixed-tracked method (FTM) developed recently can be employed to carry out the data communication in the geometry representation at each instant during numerical simulations. Therefore, the two sets of mesh-grids for the numerical simulation and the description of the moving interface of bearing are designed ([1] and Wang et al. [5, 6]). The coordinate location of the corresponding mesh grids is determined by 


$$
[\mathbf{r}]=[\mathbf{A}]^{-1}\left[\mathbf{r}^{\prime}\right]
$$

where $[\mathbf{r}]$ and $\left[\mathbf{r}^{\prime}\right]$ are the location vectors at a point on the bearing surface in the space and body coordinate systems, and the inverse Euler angular transformation in eqn. (2) is given as follows:

$$
A^{-1}=\left(\begin{array}{ccc}
\cos \beta \cos \gamma & -\sin \beta & \cos \beta \sin \gamma \\
\cos \alpha \sin \beta \cos \gamma & \cos \alpha \cos \beta & \sin \alpha \cos \beta \\
\sin \alpha \sin \beta \cos \gamma & \sin \alpha \cos \beta & \sin \alpha \sin \beta \sin \gamma+\cos \alpha \cos \gamma
\end{array}\right)
$$

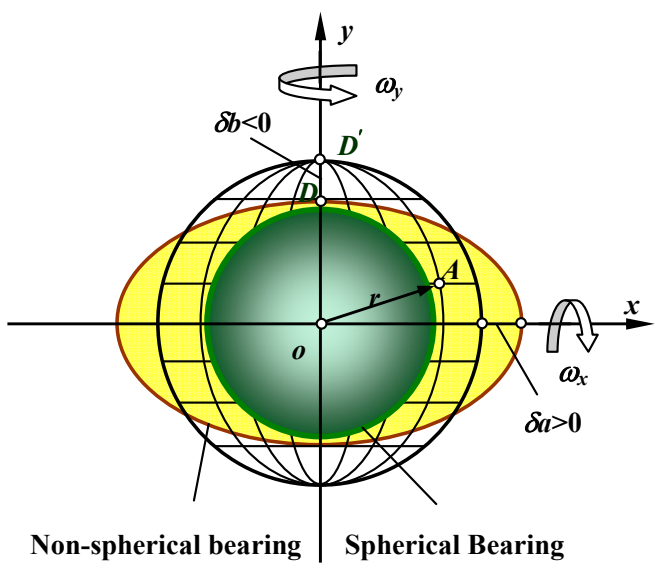

(a)

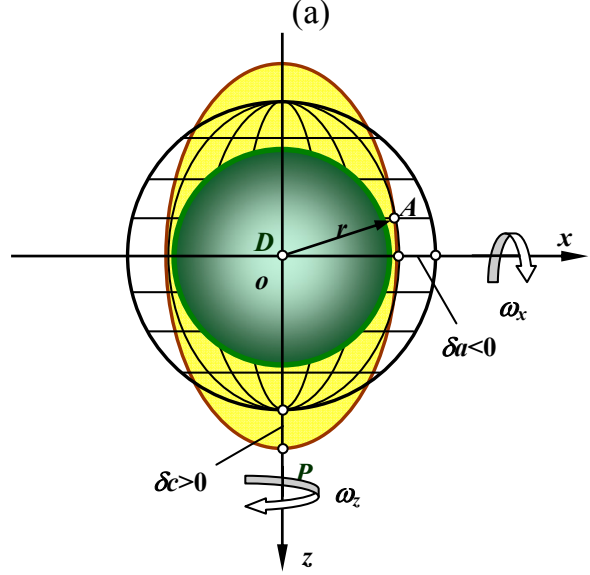

(b)

Figure 4: Variations of non-spherical bearing geometry: (a) with $\delta a$ and $\delta b$ along $\mathrm{x}$ and $\mathrm{y}$; and (b) with $\delta a$ and $\delta c$ along $\mathrm{x}$ and $\mathrm{z}$. 
where $(\alpha, \beta, \gamma)$ are three Euler angles corresponding respectively to the three Euler angular velocities $\left(\omega_{x}, \omega_{y}, \omega_{z}\right)$, as shown in fig. 2, [1, 4]. Additionally, the film thickness or gap between the bearing surfaces with the complex geometry such as non-sphericity or roughness can be described as follows [1]:

$$
\begin{aligned}
h(\varphi, \theta, t)= & \left(r_{c}-r_{b}\right)-e_{x}(t) \sin \theta \cos \varphi-e_{y}(t) \sin \theta \sin \varphi-e_{z}(t) \cos \theta \\
& +\Theta_{c b}(\varphi, \theta)+\delta_{c b}(\varphi, \theta, t)
\end{aligned}
$$

where $r_{b}$ and $r_{c}$ are the radial distance from a point on the inner or outer bearing surface of the real spherical or aspheric bearing to the centre of the nominal spherical bearing, respectively. The variables of $\left(e_{x}, e_{y}, e_{z}\right)$ are the position of the centre of the inner bearing surface with reference to the centre of the outer bearing surface. The variables of $\Theta_{c b}$ and $\delta_{c b}$ are the combined microgeometrical variation and deformation of two bearing surfaces, respectively, Wang et al. [7].

\section{Governing equation}

\subsection{Lubrication}

The lubrication problem of spherical or aspheric bearing with complex geometry includes elastohydrodynamic lubrication and mixed lubrication under the transient motion and dynamic loading condition. The lubrication film pressure can be evaluated as follows:

$$
\begin{aligned}
& \sin \theta \frac{\partial}{\partial \theta}\left(h^{3} \sin \theta \frac{\partial p}{\partial \theta}\right)+\frac{\partial}{\partial \varphi}\left(h^{3} \frac{\partial p}{\partial \varphi}\right) \\
& =6 \mu r_{c 0}^{2} \sin \theta\left[\begin{array}{l}
\left(-\omega_{x} \sin \varphi+\omega_{y} \cos \varphi\right) \frac{\partial h}{\partial \theta} \\
+\left(-\omega_{x} \cos \varphi \cos \theta-\omega_{y} \sin \varphi \cos \theta+\omega_{z} \sin \theta\right) \frac{\partial h}{\partial \varphi}
\end{array}\right] \\
& +12 \mu r_{c 0}^{2} \sin ^{2} \theta \frac{\partial h}{\partial t}
\end{aligned}
$$

where $r_{c 0}$ is the radius of the nominal spherical bearing of the bearing. For the mixed lubrication regime, the asperity contact pressure needs to be evaluated by direct contact mechanic model or other methods as described previously $[1,3,7]$.

\subsection{Friction and frictional torque}

Shear stress of fluid film of spherical or aspheric bearing dependent on velocity change of fluid film and lubricant viscosity can be expressed as follows: 


$$
[\tau]_{f}=\left\{\begin{array}{c}
\tau_{\theta \varphi} \\
\tau_{\theta r} \\
\tau_{\varphi r}
\end{array}\right\}=\left[\begin{array}{c}
0 \\
-\mu \frac{U_{\varphi}}{h}+\left(\varsigma-\frac{h}{2}\right) \frac{1}{r_{0} \sin \theta} \frac{\partial p}{\partial \varphi} \\
-\mu \frac{U_{\theta}}{h}+\left(\varsigma-\frac{h}{2}\right) \frac{1}{r_{0}} \frac{\partial p}{\partial \theta}
\end{array}\right]
$$

where $U_{\varphi}$ and $U_{\theta}$, represent the corresponding velocity components at a point on the bearing surface.

The corresponding frictional torque caused by both the fluid film shear stress and the asperity contact can be calculated by

$$
\left\{\begin{array}{l}
T_{x} \\
T_{y} \\
T_{z}
\end{array}\right\}=\iint_{S_{f}}\left[\begin{array}{c}
\left(\tau_{\theta r} \sin \varphi+\tau_{\varphi r} \cos \varphi \cos \theta\right) \\
\left.\tau_{\theta r} \cos \varphi+\tau_{\varphi r} \sin \varphi \cos \theta\right) \\
\left(\tau_{\varphi r} \sin \varphi\right)
\end{array}\right] r_{0}^{3} d \theta d \varphi+\iint_{S_{b}} p f_{c}\left[\begin{array}{c}
r_{o x} \kappa_{x} \\
r_{o y} \kappa_{y} \\
r_{o z} \kappa_{z}
\end{array}\right] r_{0}^{2} d \theta d \varphi
$$

where $\kappa_{x}, \kappa_{y}$ and $\kappa_{z}$ are equal to 1 or 0 , depending on whether there is relative motion, $f_{c}$ is the friction coefficient and $r_{o x}, r_{o y}$ and $r_{o z}$ are the corresponding arm lengths of friction stress components to the corresponding coordinate axes [3].

\subsection{Contact mechanics}

The general description for contact problem of spherical or aspheric bearing with complex geometry can be made by the restriction relationship of the gap between the two surfaces brought into contact and the corresponding contact pressure in the contact area $\Omega_{c}$ as follows:

$$
\begin{cases}g(\varphi, \theta)=0 & (\varphi, \theta) \in \Omega_{c} \\ p(\varphi, \theta)>0 & (\varphi, \theta) \in \Omega_{c} \\ g(\varphi, \theta)>0 & (\varphi, \theta) \notin \Omega_{c} \\ p(\varphi, \theta)=0 & (\varphi, \theta) \notin \Omega_{c}\end{cases}
$$

where $p$ represents contact pressure, and the gap of $g$ comprises the original unreformed gap $g_{0}$ without contact pressure and the deformation caused by contact pressure on the two surfaces ([7] and Wang et al. [8]). As an example, the contact mechanics was numerically predicted by the finite element method in this study, as shown in fig. 5, in order to validate the general methodology of data communication by the fixed-tracked method for the dynamic contact mechanics as well as the computational wear modelling.

\subsection{Computational wear prediction}

Once the contact pressure is obtained, the computational wear modelling can be carried out by the fixed-tracked method during the multi-directionally transient motion and dynamic loading condition. The wear depth evaluated by Archard's wear law, experienced an update stage with $n_{t}$ cycles, can be written as 
10 Tribology and Design

$$
\Delta r_{i}(t)=\sum_{t=1}^{n_{t}} k p_{t} s_{t}
$$

During the operating condition, the bearing geometry is modified with time due to wear. The sliding distance in spherical coordinate system is shown in fig. 6 . It should be pointed out that the modification of bearing geometry occurs on the two bearing surfaces of the bearing with a hard-on-hard material combination such as the metal-on-metal bearing [7]. The modifications of the two bearing surfaces at each instant during the operating cycle are expressed as

$$
r_{i}^{k}(t)=r_{i}^{k-1}(t) \pm \Delta r_{i}(t)
$$

where + or - sign presents the direction of modification of bearing geometry during the computational wear simulation.

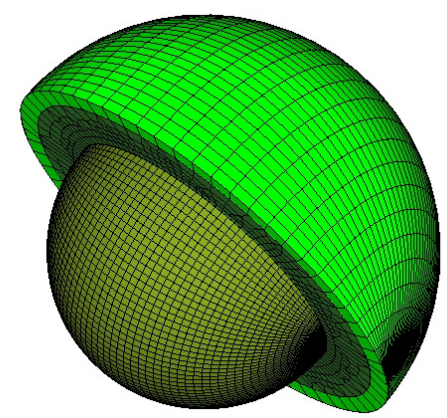

Figure 5: $\quad$ Finite element model for dynamic contact mechanics.

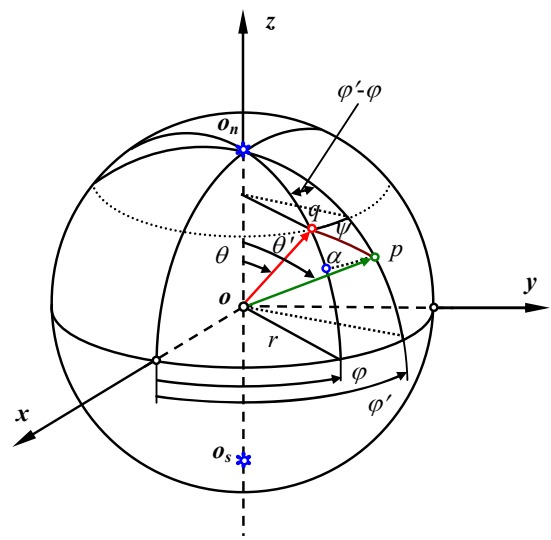

Figure 6: $\quad$ Spherical distance on bearing surface. 


\section{Numerical experiments}

The two cases of complex spherical bearings were carried out for the validation of the tribological simulation system, one for the lubrication and friction of an ellipsoidal bearing with the non-sphericity relative to the nominal spherical bearing in the $\mathrm{x}$-, the $\mathrm{y}$ - and $\mathrm{z}$-directions and the other for the computational wear prediction of a spherical bearing. The one-dimensional time-dependent motion and dynamic loading condition was employed for the study of the transient elastohydrodynamic lubrication of the non-spherical bearing. The details of the basic parameters of bearing geometry and the mechanical properties of bearing components had been reported elsewhere [1].

Firstly, the transient elastohydrodynamic lubrication of the ellipsoidal bearing geometry with the non-sphericity of $(\delta a, \delta b, \delta c)=(+6,-6,+6) \mu \mathrm{m}$ was evaluated by running the in-house software in the tribological simulation system with incorporating the general methodologies and the corresponding numerical techniques [1]. The variation of the predicted minimum film thickness with time in three operating cycles is shown in fig. 7. It was seen that the variation became convergent from the second cycles.

Additionally, the prediction of frictional torque caused by shear stress of fluid film of the non-spherical bearing with the ellipsoidal geometry was made when the lubrication result has been achieved under the same operating condition as the above-mentioned, as shown in fig. 8. It was found that the frictional torque at the instants with zero value of angular velocity became zero. This reason follows that friction only occurs in fluid film with relative velocity or velocity grade [3]. The friction stress in the mixed lubrication regime was caused by the asperity contact, which will be studied in future.

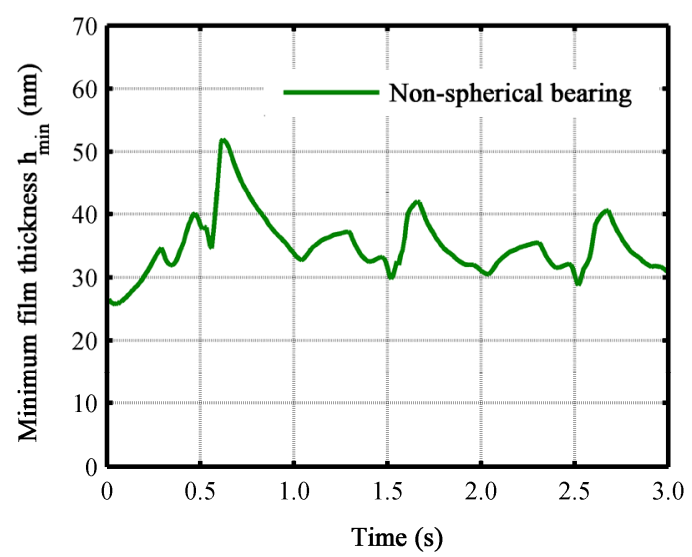

Figure 7: $\quad$ Minimum film thickness. 


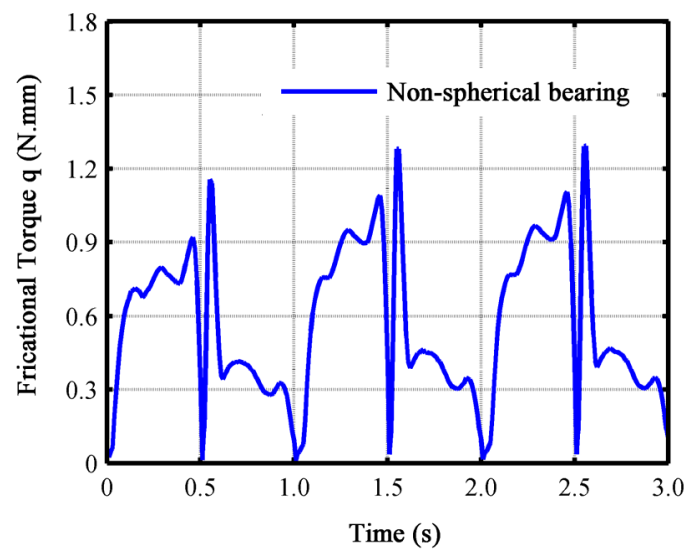

Figure 8: $\quad$ Frictional torque.

Furthermore, the computational wear prediction of metal-on-metal spherical bearing under the three-dimensional time-dependent motion and dynamic loading condition was carried out. The dynamic contact mechanics was evaluated by the tribological simulation system with the finite element model, as shown in fig. 5. The contact pressure distributions at several instant in one operating cycle were presented, as shown in figs. 9(a)-9(d). At the same time, the computational wear prediction up to 10 year ( 1 million cycles) was carried out, as shown in fig. 10. It should be pointed out that the micro contact mechanics model had to be used for wear prediction of spherical bearing with the microgeometrical variations such as roughness because of limitation of element number of the finite element method $[1,7,8]$.

\section{Conclusions}

A general tribological simulation system for the study of complex problems of spherical or aspheric bearing with the complex geometry and under the complex time-dependent motion and dynamic loading condition has been developed, in which the general methodologies and numerical techniques were incorporated on the basis of the regular spherical-grid-data-model. Two cases of an ellipsoidal bearing for the study of lubrication and friction and another spherical bearing for the study of dynamic contact mechanics and computational wear prediction under the given operating conditions were investigated. It was seen that the tribological simulation system could be employed to investigate the similar complex tribological problems of spherical or aspheric bearing with complex bearing geometry and under the complex operating condition. 
$\mathrm{t}=0.05 \mathrm{~s}$

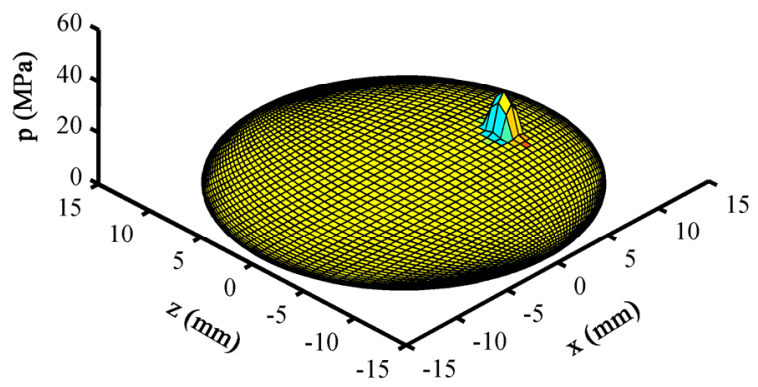

(a)

$\mathrm{t}=0.25 \mathrm{~s}$

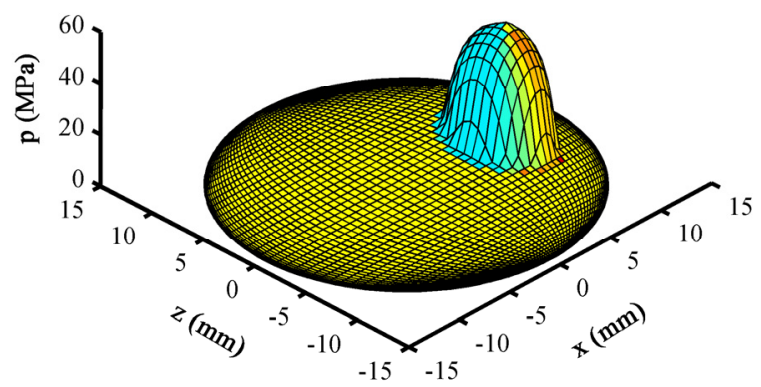

(b)

$\mathrm{t}=0.50 \mathrm{~s}$

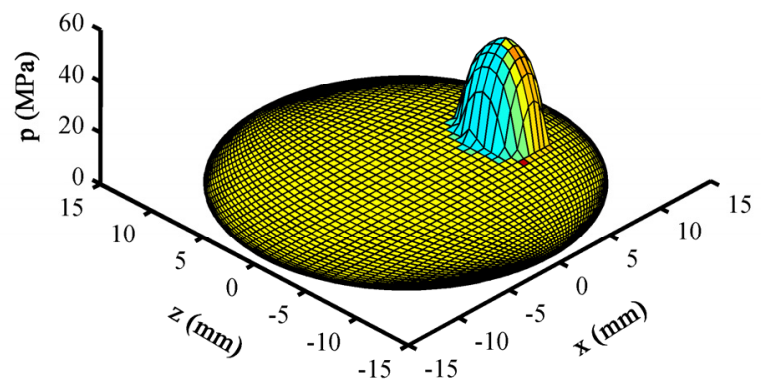

(c)

Figure 9: Dynamic contact pressures. 


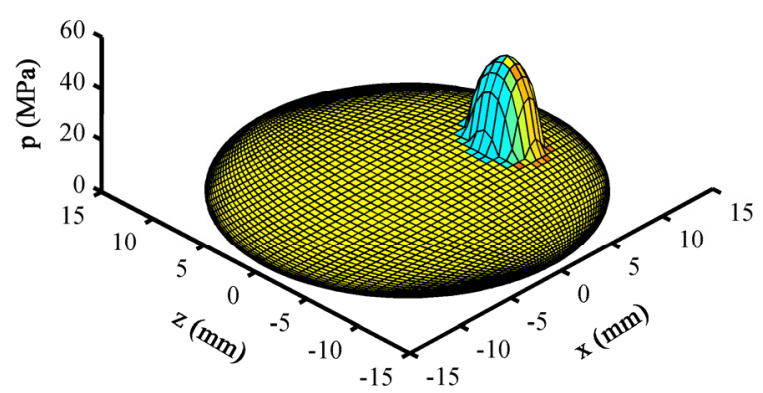

(d)

Figure 9: Continued.

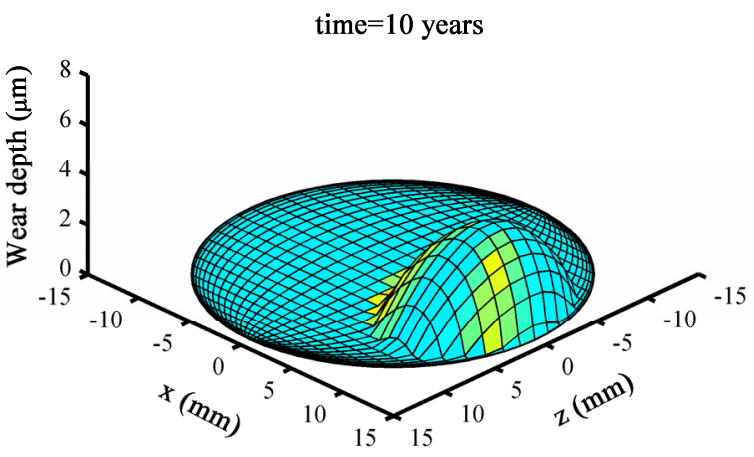

Figure 10: Computational wear prediction.

\section{Acknowledgement}

This work was supported by the National Science Foundation of China (Grant No.10972165).

\section{References}

[1] Wang, F. C., Zhao, S.X., Félix Quiñonez, A., Hu, X., Mei, X.S. \& Jin, Z., Nonsphericity of bearing geometry and lubrication in hip joint implants, ASME Journal of Tribology, 131(3), p031201, 2009.

[2] Flores, P., Ambrósio, J., Claro, J.C.P. \& Lankarani, H.M., Dynamics of multibody systems with spherical clearance joints, ASME Journal of Tribology, 1(7), pp. 240-247, 2006. 
[3] Wang, F.C, Brockett, C., Williams, S., Udofia, I.T., Fisher, J. \& Jin, Z., Lubrication and friction prediction in metal-on-metal hip Joint implants, IOP Physics in Medicine and Biology, 53, pp.1277-1293, 2008.

[4] Wang, F. C. \& Jin, Z., Transient elastohydrodynamic lubrication of hip joint implants, ASME Journal of Tribology, 130 (1), p011007, 2008.

[5] Wang, F. C., Zhao, S.X., Gao, Q.J., Fan, Q., Zeng, L.C., Félix Quiñonez, A. \& Keogh, P.S., Non-spherical bearing geometry and elastohydrodynamic lubrication of hip joint replacements under transient walking conditions, World Tribology Congress IV, Kyoto, Japan, 2009.

[6] Wang, F. C., Zhao, S.X., Xu, H., Chen, W., Mei, X.S. \& Brown, T.D., Dynamic contact mechanics and wear modelling of hip joint replacements with hard-on-hard material combination under three-dimensional Loading and transient motion, World Tribology Congress IV, Kyoto, Japan, 2009.

[7] Wang, F. C., Udofia, I.T. \& Jin, Z., An integrated experimental and theoretical contact mechanics study of UHMWPE hip implants tested in a hip simulator, ASME Proc. World Tribology Congress III, Washington, pp311-312, 2005.

[8] Wang, F. C. \& Jin. Z., Lubrication modelling of artificial hip joints, IUTAM symposium on elastohydrodynamic and micro- elastohydrodynamic 134: book series: Solid Mechanics and its Applications, Eds by Snidle RW and Evans HP, Springer, pp385-396, 2006. 